\title{
The epidemiology and incidence of dengue in Makkah, Saudi Arabia, during 2017-2019
}

Sami Melebari, MSc, PhD, Rowaida Bakri, MSc, PhD, Abdul Hafiz, MSc, PhD, Fadel Qabbani, BMSc, Asim Khogeer, MSc, PhD, IsmailAlharthi, BMSc, Sultan Alhazmi, MPh, Yehya Almalki, BMSc, Rasha Bulkhi, MSc, Razaz Gammash, MSc, Ashwaq Hakim, BMSc, Ahmed Alkhyami, BMSc, Mohamed Bazaid, MSc, Thanaa Mohammad, MBBS, MD.

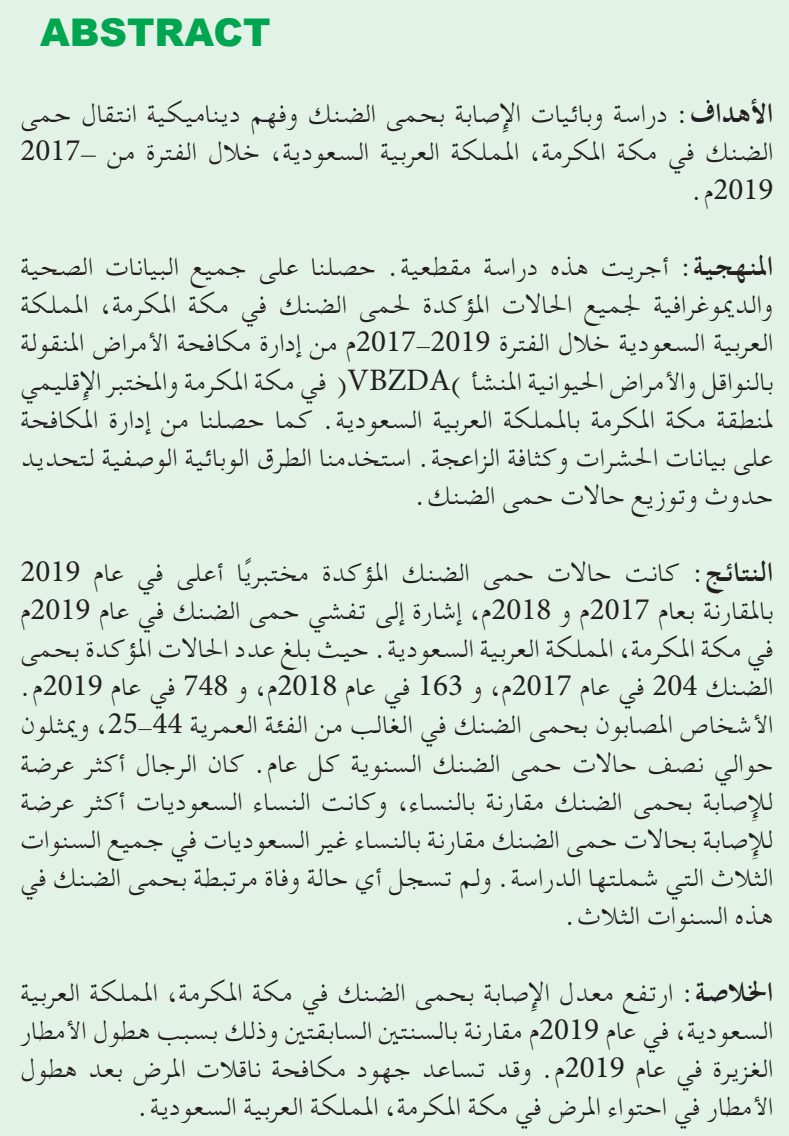

Objectives: To study the epidemiology of dengue incidence and understand the dynamics of dengue transmission in Makkah, Kingdom of Saudi Arabia (KSA), between 2017-2019.

Methods: This is a cross-sectional study. Health and demographic data was obtained for all confirmed dengue cases in Makkah, KSA, in the years 2017-2019 from the Vector-Borne and Zoonotic Diseases Administration (VBZDA) in Makkah and the Makkah Regional Laboratory, KSA. In addition, entomological data about Aedes density was obtained from the VBZDA. Descriptive epidemiological methods were used to determine the occurrence and distribution of dengue cases.
Results: Laboratory-confirmed dengue cases were higher in 2019 as compared to 2017 and 2018, suggesting an outbreak of dengue in Makkah, KSA, in 2019. The incidence of confirmed dengue cases was 204 in 2017, 163 in 2018 and 748 in 2019. Dengue mostly affected people in the 25-44 age group, accounting for approximately half of the annual dengue cases each year. Men were at a higher dengue incidence risk when compared to women, and Saudi women had a higher risk rate for dengue cases when compared to non-Saudi women in all 3 years studied. There was no dengue related death in these 3 years.

Conclusion: The dengue incidence increased in Makkah, KSA, in 2019 as compared to the previous 2 years, owing to heavy rainfall in 2019. Post-rainfall Vector control efforts may help contain the disease in Makkah, KSA.

Keywords: Dengue, incidence, Vector Borne diseases, Saudi Arabia

Saudi Med J 2021; Vol. 42 (11): 1173-1179 doi: 10.15537/smj.2021.42.11.20210124

From the Department of Molecular Biology (Melebari, Qabbani, Hakim, Bazaid); from the Department of Microbiology (Alharthi, Bulkhi, Gammash, Alkhyami); from the Department of Blood Bank Centre (Mohammad), The Regional Laboratory; from the Department of Research and Planning (Khogeer), General Directorate of Health Affairs Makkah Region; from the Vector-Borne and Zoonotic Diseases Administration (Alhazmi, Almalki), Public Health, Ministry of Health, and from the Department of Medical Parasitology (Bakri, Hafiz), College of Medicine, Umm Al Qura University, Makkah, Kingdom of Saudi Arabia.

Received 1st April 2021. Accepted 1st September 2021.

Address correspondence and reprint request to: Dr. Abdul Hafiz, Department of Medical Parasitology, College of Medicine, Umm Al Qura University, Makkah, Kingdom of Saudi Arabia. E-mail:aaahafiz@uqu.edu.sa

ORCID ID: https://orcid.org/0000-0002-1898-8841 
$\mathrm{D}$ engue fever (DF) is one of the most serious re-emerging infectious diseases in the world. The disease, in recent decades, has expanded in its geographical distribution and becomes endemic in more than 100 countries, with around 2.5 billion people at risk of infection. ${ }^{1}$

The World Health Organization (WHO) estimates that there could be over 50 million incidences of DF each year worldwide. ${ }^{2,3}$ The causes for the appearance of this disease are not fully understood. However, changes in the socio-demographic and public health infrastructures of affected countries over the past decades might have significantly contributed to the changing dynamics of dengue incidence. ${ }^{4}$

The WHO reports that the dengue virus can cause classic DF, dengue shock syndrome (DSS) and dengue hemorrhagic fever. Dengue fever is an arthropod Vector-Borne disease that is spread by different species of biting female Aedes (Ae.) mosquitos (Ae. aegypti; Ae. albopictus; Ae. polynesiensis). Aedes aegypti is the more important vector because it exists in proximity to human habitation. ${ }^{5}$

The dengue viruses belong to the genus of Flavivirus and family of Flaviviridae. ${ }^{6}$ It has genetic material composed of single-strand ribonucleic acid (RNA) and has 4 distinct serotypes dengue virus (DENV 1-4). ${ }^{7}$ The virus is found in tropical and sub-tropical climates worldwide with high humidity and hot temperatures, and generally in urban and semi-urban locations. ${ }^{8}$ High humidity lengthens the mosquito's lifespan, as well as minimizes the time required for viral replication. After being ingested by the mosquito host, the virus may require 3-14 days of incubation period in mosquito to transmit to a new human host. This incubation period is lesser in high humidity environments. ${ }^{9}$

In Kingdom of Saudi Arabia (KSA), in 1994, dengue virus serotype 2 (DENV-2) was recorded for the first time, and was responsible for a major epidemic in Jeddah, KSA, with 289 confirmed cases reported. ${ }^{10}$ Since then, dengue cases have been reported in other geographical locations of KSA.

In Makkah, KSA, the number of dengue cases has been reported as dengue-endemic in the last decade. In 2004, dengue case profiles were initially reported for hospitalized patients at a single center, during the first outbreak of dengue in Makkah. A total of 159 DF cases were admitted to a tertiary hospital in Makkah from

Disclosure. Authors have no conflict of interests, and the work was not supported or funded by any drug company.
2006-2008, and $77 \%$ of these cases were during the spring and early summer. Later on, a massive increase in construction work in Makkah between 2008-2012 raised the number of DF, with 4,187 cases reported, due to the rise in stagnant water.$^{11-13}$

Many factors are further related to increasing the spread of this disease, including growing levels of urbanization, travel, international trade, climate change and poor vector control. In addition, irregular insecticide spray, development of insecticide resistance in mosquitos and changing climate conditions may contribute to the outbreaks of the disease. ${ }^{14}$

Prevention and control of dengue disease are still the most substantial steps needed to minimize the danger of infection. Understanding the disease's prevalence and distribution over time are important if we are to effectively administer interventional measures. Therefore, we carried out this study to determine the epidemiology of dengue spread in Makkah between 2017-2019.

Methods. This cross-sectional study recruited laboratory-confirmed dengue cases in Makkah hospitals, KSA, between January 2017 and December 2019. The study subjects were mostly citizens and residents of Makkah. However, visitors with confirmed dengue cases were also included in this study. All blood samples were collected within 5-10 days of illness onset. The diagnosis of the disease was made by the regional laboratory in Makkah. The results were recorded on the Health Electronic Surveillance Network (HESN) website according to the system of the Ministiry Of Health $(\mathrm{MOH})$. Patients with negative results were excluded from the study.

The inclusion criteria was citizen, resident, or visitor to Makkah city with laboratory confirmed dengue, during the study period. The exclusion criteria was citizen, resident, or visitor to Makkah city who did not test positive for dengue infection during the study period and all who were not citizen, resident, or visitor of Makkah city during the study period.

All suspected dengue disease samples were sent upon collection to the regional laboratory from different hospitals in Makkah to detect the dengue virus to confirm the diagnosis. The samples were stored at $-30^{\circ} \mathrm{C}$ under consistent frozen situations for more molecular assays.

Makkah is the ancient Holy City of the islamic world and the capital of Makkah Province. It is situated in the western part of KSA, at a longitude of 39.826168 East and a latitude of 21.422510 North. The city is at an elevation of $277 \mathrm{~m}$ (909 ft) above sea level and lies approximately $70 \mathrm{~km}$ (44 mi) inland from Jeddah on 
the Red Sea. The estimated population in Makkah was more than 2 million in 2020, making it the third-most populated city in Saudi Arabia. ${ }^{15}$ Raising that figure, every year, approximately 2 million Haj pilgrims and 6-7 million Umrah pilgrims visit and stay in Makkah, KSA, coming from different parts of the world. ${ }^{16,17}$ Makkah's climate is hot in summer, with temperatures often more than $40^{\circ} \mathrm{C}\left(104^{\circ} \mathrm{F}\right)$ during the day and dropping only to $30^{\circ} \mathrm{C}\left(86^{\circ} \mathrm{F}\right)$ at night. The climate is then moderate in winter, with temperatures of around $30^{\circ} \mathrm{C}\left(86^{\circ} \mathrm{F}\right)$ in the day and $18^{\circ} \mathrm{C}\left(64^{\circ} \mathrm{F}\right)$ at night; there is little rainfall in Makkah between November and January. ${ }^{18}$

Prior to designing the study, a through literature search was conducted to review all the published research article on dengue incidence in Makkah region, KSA. For this, PubMed search engine from the National Library of Medicine, National Centre for Biotechnology Information was used. All published articles we obtained were analyzed.

Ethical approval was obtained from the Ethics Committee of the $\mathrm{MOH}, \mathrm{KSA}$ (ethical approval reference no. H-02-K-076-0420-287). Data was collected with prior approval to conduct the study from the MOH. Patients' data collected from the regional laboratory and the Vector-Borne and Zoonotic Diseases Administration in Makkah were anonymized and analyzed for this study. The study did not directly involve any human subject, laboratory data of human subjects were obtained after ethical approval.

RNA extraction. Extraction from $300 \mu \mathrm{l}$ of the serum sample was carried out using the MagNA Pure 96 instrument (Roche Molecular Systems, Inc., Germany), following the manufacturer's instructions. ${ }^{19}$ All chemicals and equipment used in this study were supplied by the $\mathrm{MOH}$.

RT-PCR assay. Reverse transcription polymerase chain reaction (RT-PCR) was carried out using a LightMix Modular Dengue Virus kit. $10 \mu \mathrm{l}$ of the reaction mix was combined with $10 \mu \mathrm{l}$ of the extracted RNA, following the manufacturer's instructions. The PCR reaction per well consisted of $4.0 \mu \mathrm{l}$ Roche master, $0.1 \mu \mathrm{l}$ RT enzyme, $0.5 \mu \mathrm{l}$ reagent mix containing primers and probes and $5.4 \mu \mathrm{l} \mathrm{PCR}$-grade water. The step of reverse transcription was achieved via the following technique: $55^{\circ} \mathrm{C}$ RT step for 5 minutes, $95^{\circ} \mathrm{C}$ denaturation for 5 minutes, 45 cycles of 5 seconds at $95^{\circ} \mathrm{C}, 15$ seconds at $60^{\circ} \mathrm{C}$ then 15 seconds at $72^{\circ} \mathrm{C}$ in quantification mode and, finally, cooling at $40^{\circ} \mathrm{C}$ for 30 seconds. All procedures were carried out in a LightCycler 480 instrument and data was investigated with LightCycler software 480 (Roche Molecular Systems, Inc., Germany). ${ }^{20}$

Mosquito counting and density calculation. Mosquito samples were collected by field teams and then sent to the laboratory to determine types and numbers. Density was calculated by averaging, for all the sites explored, the number of mosquitos per room.

Statistical analysis. Data was inputted into Microsoft Excel sheets. Data entries were double-checked to rule out potential errors in the data entry. Microsoft Excel was used for all data analysis, as well as plotting curves for data display. Prevalence and incidence rates were calculated as previously described. ${ }^{21}$ Pearson correlation coefficients were calculated to determine whether or not there was rainfall using Microsoft Excel.

Results. The suspected and confirmed dengue cases in Makkah, KSA, were compared in 2017, 2018 and 2019. A total of 1,004 in 2017, 752 in 2018 and 1571 in 2019 blood samples from suspected dengue cases from Makkah hospitals were sent for dengue diagnosis in the laboratory. The diagnostic test positive rate for dengue in Makkah was more (47.6\%) in 2019 as compared to 2017 and 2018 (Table 1).

There was a marked increase in dengue cases in Makkah during 2019 when compared to 2017 and 2018. Figure 1 displays the total laboratory-confirmed dengue cases and the cumulative percentage of confirmed dengue cases in Makkah by week, for 2017$2019 .{ }^{22}$ In 2019, the confirmed dengue cases were high when compared to 2017 and 2018. An early onset of dengue cases was observed in 2019 when compared to the previous 2 years. Half of the total annual dengue cases were reported by week 18 in 2017, by week 23 in 2018 and by week 13 in 2019, suggesting there was a shift in yearly dengue infection dynamics. In 2018 and 2019, in addition to high cases of dengue in the first half of the year, the dengue cases tended to rise again at

Table 1 - Average annual rainfall and dengue cases in Makkah, Kingdom of Saudi Arabia.

\begin{tabular}{lcccc}
\hline Year & Annual rainfall $(\mathrm{mm})$ & Suspected dengue cases & Confirmed dengue cases & The precentage of suspected cases found to be positive \\
\hline 2017 & 25.15 & 1,004 & 204 & $20.32 \%$ \\
2018 & 11.74 & 752 & 163 & $21.67 \%$ \\
2019 & 110.8 & 1,571 & 748 & $47.61 \%$ \\
\hline
\end{tabular}


the end of the year. No dengue deaths were reported in Makkah in any of the 3 study years.

An analysis of the density of Aedes mosquitoes in Makkah reveals that the mosquito density was higher in 2019 when compared to 2017 and 2018, as shown in Figure 2. The average observed density of Aedes mosquitos was 0.04 mosquitoes per room in 2017, 0.06 mosquitoes per room in 2018 and 0.09 mosquitoes per room in 2019. Monthly analysis of Aedes densities reveals that, for the first 10 months of the year, the Aedes density in 2017 was slightly higher when compared to 2018. However, there was a marked increase in Aedes density in November and December 2018.

When analyzed for age distribution, the highest cases were reported in the $25-44$ age group (Figure 3), which accounted for almost $50 \%$ of the dengue cases in all 3 years. Children younger than one year old least contributed to dengue cases. The highest contributing group for dengue cases (25-44 years) was responsible for confirmed 176 dengue cases in 2017, 72 cases in 2018 and 383 cases in 2019.

An analysis of dengue cases based on gender and nationality reveals that, in Makkah, the dengue incidence rate was higher for males when compared to females, within both Saudi and non-Saudi groups, for all 3 years (Figure 4). In 2017, the incidence risk of dengue infection was almost equal in Saudi males (23.54 cases per 100,000 population) and non-Saudi males (22.44 cases per 100,000 population). In 2018, the incidence risk for dengue infection was higher in Saudi males (12.70 cases per 100,000 population) than non-Saudi males (8.81 cases per 100,000 population). The incidence risk for dengue infection in 2019 was higher in non-Saudi males (59.34 cases per 100,000 population) than Saudi males ( 40.16 cases per 100,000 population). The incidence risk for dengue infection remained high for Saudi women when compared to non-Saudi women in all the 3 years studied. a

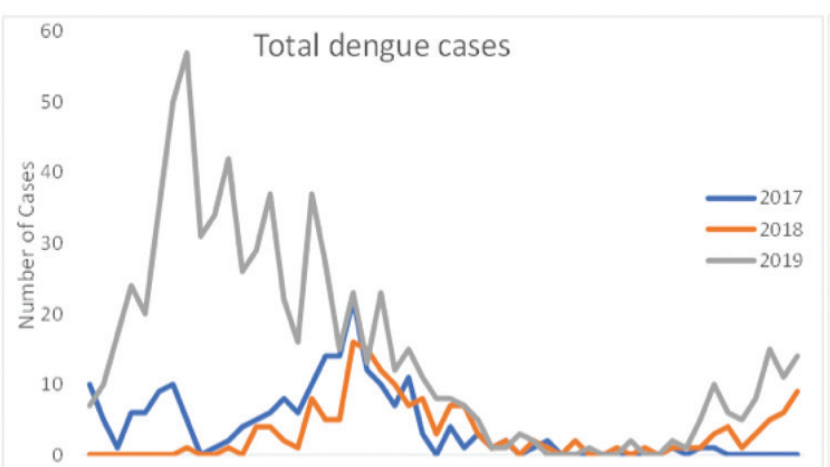

b

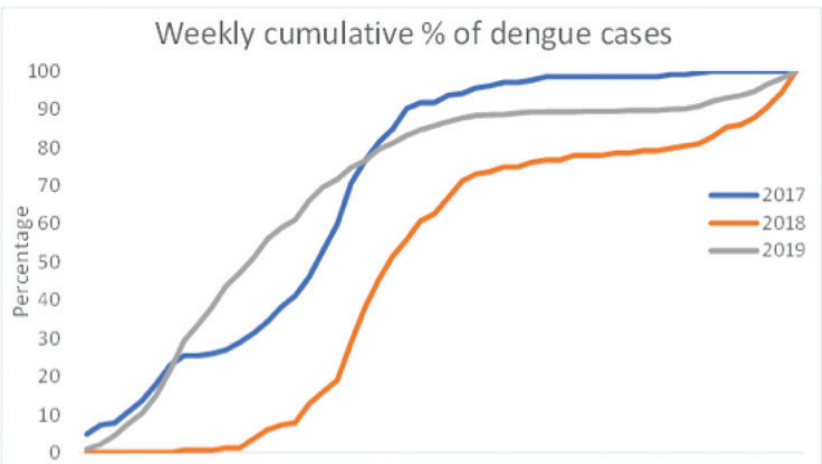

Figure 1 - Dengue cases in Makkah, Kingdom of Saudi Arabia, by weeks. (A) The total number of confirmed dengue cases was plotted against the week number in 2017, 2018 and 2019. (B) Cumulative percentages of the total number of confirmed dengue cases were plotted against the week number in 2017, 2018 and 2019.

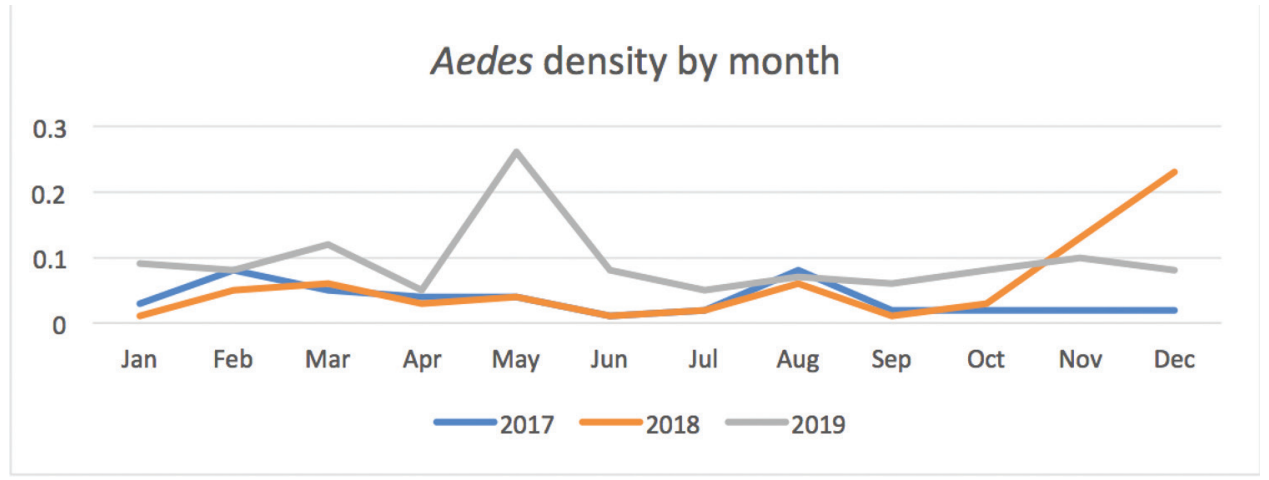

Figure 2 - Aedes density by year in Makkah, Kingdom of Saudi Arabia. The Aedes density is plotted against the months of the year in 2017,2018 and 2019. 


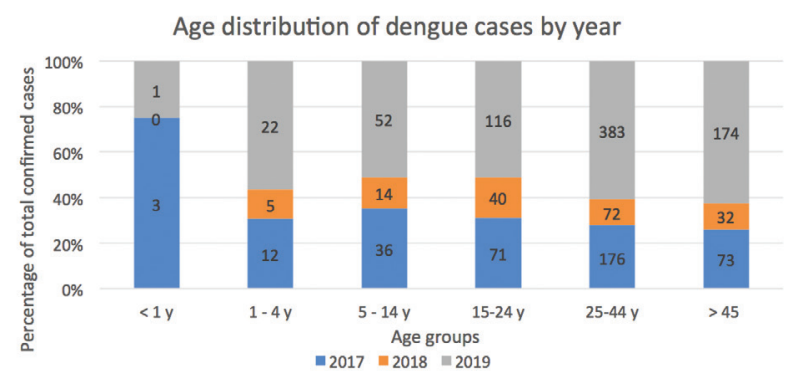

Figure 3 - Age distribution of dengue cases in Makkah, Kingdom of Saudi Arabia. The dengue cases in an age group are displayed as a percentage of the total. Each bar is divided into different colored segments to represent the proportion of dengue cases in 2017, 2018 and 2019 for each age group.

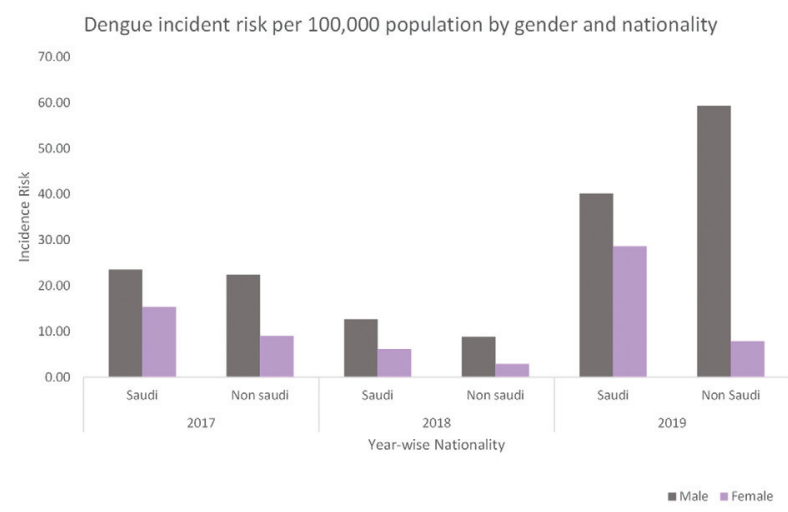

Figure 4 - Nationality and gender-related incidence risk of dengue cases in Makkah, Kingdom of Saudi Arabia, plotted from 20172019.

Discussion. Global dengue infections are on the rise. In 2000, a total of 505,403 dengue cases were reported, as compared to 2.4 million dengue cases in 2010 and 4.2 million in 2019. There were 960 denguerelated deaths reported in 2000, as compared to 4,032 in 2015 (WHO).

The weekly dynamics of dengue cases were quite different in the 3 years studied in Makkah, KSA. Notably, in 2019, 50\% of the annual dengue cases were reached by week 13, while it was week 23 in 2018 and week 18 in 2017. The dynamics of dengue cases appear to be related to rainfall, as illustrated in Table 1 . In 2017, approximately half of the annual rainfall was received by the end of February. However, in 2018, approximately $80 \%$ of the annual rainfall was received in October and November.

There was a sharp increase in Aedes density at the end of 2018. In fact, this rainfall at the end of 2018 might have contributed to the early onset of the dengue peak in 2019. The known dengue vectors are female Aedes mosquitoes belonging to 2 species, Aedes aegypti and Aedes albopictus. The latter has not been reported to be present in the western part of KSA; therefore, Aedes aegypti is thought to be the principal vector for dengue spread in western KSA. Thus, Aedes density impacts the dengue incidence. However, the methods used to measure Aedes density may give different results. In our study, we included Aedes density as calculated around the homes of confirmed dengue cases. Samples were collected from 10 houses in each direction around the house of a confirmed dengue patient. This method has a flaw in cases where the dengue infection has been acquired at another location. For instance, a dengue infection acquired at a workplace or a picnic spot may not be reflected well in mosquito density, if we calculate mosquito density around the residence of the patient. Moreover, patients' vulnerability to mosquito bites may be different in the same locality, as some people live in well-protected environments while others do not.

Dengue cases were more concentrated in the age group of 15 years or above. This suggests that the dengue infection is mainly acquired through outdoor biting mosquitoes, as small children and women contribute to lesser cases in the city. The outdoor employment of the adult population may expose them to a greater risk of dengue infection. Moreover, little immunity has been acquired in the population against the symptomatic infection since the incidences of dengue are few. It would be interesting to study how immunity to the dengue virus develops and lasts in the Saudi population. Since our study focused only on symptomatic patients who came to the hospital for treatment, this study cannot answer questions on immunity in the population. Studies that measure symptomatic and asymptomatic dengue infections to characterize age-dependent dengue immunity in the population are urgently needed, given the alarming rise of dengue cases at local and global levels.

We observed differences in the reported dengue cases in males and females. There are several infectious and non-infectious diseases where the 2 genders behave differently. When it comes to dengue, males were consistently higher in reporting confirmed cases in Makkah. The incidence rate of confirmed dengue cases was higher among males when compared to women in all 3 years studied. There can be multiple explanations for this observation; the biological susceptibility to dengue or severity of the dengue disease may be different in males and females. There may also be differences in reporting the illness or in health-seeking behavior in the 
different genders. Alternatively, the site of work may have exposed males to a greater risk of mosquito bites when compared to females. Women are less likely to be employed in remote areas where mosquito densities may be higher. There were differences in the dengue cases between Saudi citizens and foreigners in Makkah. In 2017-2018, Saudis reported more dengue cases when compared to foreigners in Makkah. This may be due to the free availability of healthcare to Saudi citizens when compared to the foreigners, all of whom may not have free access to healthcare. However, in 2019, more non-Saudi males reported dengue cases when compared to Saudi males in Makkah. This may be due to the work-related exposure of non-Saudis in 2019, a year of heavy rain. Moreover, since there was a global increase in dengue cases, visitors may have contributed more to dengue cases in 2019 as compared to the previous 2 years. Since there are more male pilgrims visitors to Makkah as compared to female pilgrim visitors, the foreigner females may thus have contributed fewer cases when compared to Saudi females in 2019. There were no dengue-related deaths reported during our study period in Makkah.

Study limitations. There are limitations to our study that should be considered when interpreting the results. Since dengue severity is not recorded at the VectorBorne disease unit (VBDU) and the regional laboratory of Makkah, KSA, this study could not examine the epidemiology of the different severities of dengue cases. The incidence rate calculations were based on the 2010 census. We could not calculate incidence rates in different age groups since the national census did not provide population data by age group in Makkah. Furthermore, asymptomatic infections and patients who did not report to healthcare units in Makkah were missed in this study. Makkah has a very dynamic population, with annual visitors numbering more than 4 times its resident population; for this reason, the incidence rate calculations should be interpreted with caution.

The study calls for renewed efforts on dengue control programs in Makkah. The increase in dengue cases with annual rainfall suggests that good mosquito control programs may help to contain dengue outbreaks. The changing pattern of rainfall demands that future civil infrastructure should be designed to minimize water clogging and the creation of mosquito breeding sites. Integrative approaches to vector control programs may be adopted. We suggest that, after each major rainfall in a locality, local vector control teams should be activated to insecticide spray hotspot areas. Water samples from potential mosquito breeding sites should be collected for at least 2 weeks following major rainfall to determine the presence of mosquito larvae. Appropriate larvicidal measures can then be employed to control mosquito breeding. Community education campaigns about water clogging and mosquito breeding should also be increased. Since Makkah receives visitors from all over the world, potentially, this city could receive global dengue genotypes. Different dengue types have been associated with different clinical manifestations of the disease. ${ }^{21}$ As such, we recommend that the $\mathrm{MOH}$ includes the detection of dengue type in its PCR diagnosis protocol. More studies are needed to characterize the epidemiological, environmental, clinical, immunological and molecular aspects of dengue in Makkah.

In conclusion, this study reports that there was a dengue outbreak in Makkah during 2019. Effective vector control measures following a rainfall may help control dengue in Makkah.

Acknowledgment. The authors gratefully acknowledge the Ministry of Health in KSA for providing data and support. We also would like to thank Cambridge Proofreading LLC, Chicago, Illinois, USA. (https://proofreading.org/) for English language Editing.

\section{References}

1. Gubler DJ. The global emergence/resurgence of arboviral diseases as public health problems. Arch Med Res 2002; 33: 330-342.

2. Gubler DJ. Dengue and dengue hemorrhagic fever. Clin Microbiol Rev 1998; 11: 480-496.

3. Gibbons RV, Vaughn DW. Dengue: an escalating problem. BMJ 2002; 324: 1563-1566.

4. Gubler DJ. The global pandemic of dengue/dengue haemorrhagic fever: current status and prospects for the future. Ann Acad Med Singap 1998; 27: 227-234.

5. Calisher CH, Karabatsos N, Dalrymple JM, Shope RE, Porterfield JS, Westaway EG, et al. Antigenic relationships between flaviviruses as determined by cross-neutralization tests with polyclonal antisera. J Gen Virol 1989; 70: 37-43.

6. Khan NA, Azhar EI, El-Fiky S, Madani HH, Abuljadial MA, Ashshi AM, et al. Clinical profile and outcome of hospitalized patients during first outbreak of dengue in Makkah, Saudi Arabia. Acta Trop 2008; 105: 39-44.

7. Gubler DJ, Ooi EE, Vasudevan S, Farrar J. Dengue and dengue hemorrhagic fever [Internet]. CABI; 2014 [2021 March 03]. Available from: https://www.cabi.org/cabebooks/ ebook/20143309279

8. Simmons CP, Farrar JJ, van Vinh Chau N, Wills B. Dengue. $N$ Engl J Med 2012; 366: 1423-1432.

9. Alhaeli A, Bahkali S, Ali A, Househ MS, El-Metwally AA. The epidemiology of dengue fever in Saudi Arabia: a systematic review. J Infect Public Health 2016; 9: 117-124.

10. Fakeeh M, Zaki AM. Virologic and serologic surveillance for dengue fever in Jeddah, Saudi Arabia, 1994-1999. Am J Trop Med Hyg 2001; 65: 764-767. 
11. Gubler DJ. Epidemic dengue/dengue hemorrhagic fever as a public health, social and economic problem in the 21 st century. Trends Microbiol 2002; 10: 100-103.

12. Shahina W, Nassara A, Kalkattawia M, Bokharia H. Dengue fever in a tertiary hospital in Makkah, Saudi Arabia [Internet]. WHO Regional Office for South-East Asia; 2009 [accessed 2021 February 25]. Available from: https://apps.who.int/iris/ handle/10665/170727

13. Alwafi O, McNabb S, Memish Z, Assiri A, Alzahrani $S$, Asiri $S$, et al. Dengue fever in Makkah, Kingdom of Saudi Arabia, 2008-2012 [Internet]. American Journal of Research Communication; 2013 [accessed 2021 March 3]. Available from: http://www.usa-journals.com/wp-content/ uploads/2013/10/alwafi_Vol111.pdf

14. Alshamrani S, Assiri A, Bin Saeed A, Alhakeem R, McNabb SJN. Distribution and determinants of dengue fever, cities of Jeddah and Makkah, Kingdom of Saudi Arabia, 2007-2013 [Internet]. EMORY; 2013 [Accessed 2017 September 2]. Available from: http://kingabdullahfellowship.com/wp-content/uploads/ Sultan-Alshamrani-Poster.pdf

15. Altassan KK, Morin C, Shocket MS, Ebi K, Hess J. Dengue fever in Saudi Arabia: a review of environmental and population factors impacting emergence and spread. Travel Med Infect Dis 2019; 30: 46-53.

16. Mecca, Saudi Arabia metro area population 1950-2021 [Internet]. macrotrends; 2021 [2021 March 03]. Available from: https://www.macrotrends.net/cities $/ 22426 /$ meccal population
17. Open data platform [Internet]. Ministry of Hajj and Umrah [2021 March 04]. Available from: https://www.haj.gov.sa/en/ InternalPages/Details/54

18. Mecca weather and temperature [Internet]. the Bahrain Property Site [2021 March 03]. Available from: http:// thebahrainpropertysite.com/mecca-weather.html

19. High-throughput detection of bacterial fungal and viral nucleic acids in routine microbiological sample types using one generic Pathogen Universal Protocol on the MagNA Pure 96 System [Internet]. Regensburg: Roche; 2015 [2021 March 01]. Available from: https://lifescience.roche.com/content/ $\mathrm{dam} / \mathrm{RMS} /$ lifescience/Documents/PDF/High-throughputdetection-of-bacterial-fungal-and-viral-nucleic-acids-inroutine-microbiological-sample-types.pdf

20. Tsai HP, Tsai YY, Lin IT, Kuo PH, Chang KC, Chen JC, et al. Validation and application of a commercial quantitative real-time reverse transcriptase-PCR assay in investigation of a large dengue virus outbreak in Southern Taiwan. PLoS Negl Trop Dis 2016; 10: e0005036.

21. Yung CF, Lee KS, Thein TL, Tan LK, Gan VC, Wong JGX, et al. Dengue serotype-specific differences in clinical manifestation, laboratory parameters and risk of severe disease in adults, Singapore. Am J Trop Med Hyg 2015; 92: 999-1005.

22. Sanna M, Hsieh YH. Temporal patterns of dengue epidemics: the case of recent outbreaks in Kaohsiung. Asian Pac J Trop Med 2017; 10: 292-298. 